\title{
Uncertainty in Sequential Pattern Mining
}

\author{
Muhammad Muzammal and Rajeev Raman \\ Department of Computer Science, University of Leicester, UK. \\ \{mm386, r.raman\}@mcs.le.ac.uk
}

\begin{abstract}
We study uncertainty models in sequential pattern mining. We discuss some kinds of uncertainties that could exist in data, and show how these uncertainties can be modelled using probabilistic databases. We then obtain possible world semantics for them and show how frequent sequences could be mined using the probabilistic frequentness measure.
\end{abstract}

Key words: Mining Uncertain Data, Sequential Pattern Mining, Probabilistic Databases, Theoretical Foundations of Data Mining.

\section{Sequential Pattern Mining}

Sequential pattern mining [2] is an important data mining problem: it is concerned with databases that contain sequences of events, each of which is associated with a source. For example, a transaction database of a store may contain sequences of purchases (events) made by individual customers (sources), and the objective is to find patterns of customer purchasing behaviour in successive visits. This has applications in various domains including transaction databases, web access patterns and biological sequences, and is formally defined as follows. Let $\mathcal{I}=\left\{i_{1}, i_{2}, \ldots, i_{q}\right\}$ be a set of items and $\mathcal{S}=\{1, \ldots, m\}$ be a set of sources. An event $e \subseteq \mathcal{I}$ is a collection of items. A database $D=\left\langle r_{1}, r_{2}, \ldots, r_{n}\right\rangle$ is an ordered list of records such that each $r_{i} \in D$ is of the form $\left(e i d_{i}, e_{i}, \sigma_{i}\right)$, where $e i d_{i}$ is event-id, $e_{i}$ is an event and $\sigma_{i}$ is a source. A sequence $s=\left\langle s_{1}, s_{2}, \ldots, s_{a}\right\rangle$ is an ordered list of events. Let $s=\left\langle s_{1}, s_{2}, \ldots, s_{q}\right\rangle$ and $t=\left\langle t_{1}, t_{2}, \ldots, t_{r}\right\rangle$ be two sequences. We say that $s$ is a subsequence of $t$, denoted $s \preceq t$, if there exist integers $1 \leq i_{1}<i_{2}<\cdots<i_{q} \leq r$ such that $s_{k} \subseteq t_{i_{j}}$, for $k=1, \ldots, q$. The source sequence corresponding to a source $i$, denoted by $D_{i}$, is just the multiset $\{e \mid(e i d, e, i) \in D\}$, ordered by eid. For a sequence $s$ and source $i$, let $X_{i}(s, D)$ be an indicator variable, whose value is 1 if $s \preceq D_{i}$, and 0 otherwise. The objective is to find all sequences $s$ whose support (Supp) is at least some user-defined threshold $\theta, 1 \leq \theta \leq m$, where $\operatorname{Supp}(s, D)=\sum_{i=1}^{m} X_{i}(s, D)$.

\section{Modelling Uncertainty}

Traditionally, it is assumed that data is deterministic. However, it is now recognized that data is often inherently noisy or uncertain. Probabilistic databases are one way to model such uncertainties $[1,7]$. Recently, many data mining problems 
have been studied in probabilistic databases including frequent itemset mining [1, $3]$. We focus on sequential pattern mining and our interest is in situations where there is uncertainty either about a source or in the associated events.

Source-Level Uncertainty. In a retail transaction database, a customer's details may be incomplete or incorrect, or the database may itself be uncertain as a result of "deduplication" or cleaning [4], leading to ambiguity in the customer's identity. A person/vehicle may be detected by a sensor/camera, but identification methods may be noisy, leading to uncertainty (take the UK police's automatic number plate recognition database [9] for example). In such scenarios, it is certain that an event occurred (e.g. a customer bought some items, a vehicle/person entered an area) but there is uncertainty about the source associated with that event. Situations like this can be modelled using attribute level uncertainty [7], when the 'source' attribute is a probability distribution over sources.

A probabilistic database $D^{p}$ is an ordered list $\left\langle r_{1}, \ldots, r_{n}\right\rangle$ of records of the form (eid,e,W) where eid is an event-id, $e$ is an event and $W$ is a probability distribution over $\mathcal{S}$. The distribution $W$ contains pairs of the form $(\sigma, c)$, where $\sigma \in \mathcal{S}$ and $0<c \leq 1$ is the confidence that the event $e$ is associated with source $\sigma$; we assume $\sum_{(\sigma, c) \in W} c=1$. A possible world $D^{*}$ of $D^{p}$ is generated by taking each event $e_{i}$ in turn, and assigning it to one of the possible sources $\sigma_{i} \in W_{i}$, where $\sigma_{i} \in \mathcal{S}$. Thus every record $r_{i}=\left(\right.$ eid $\left._{i}, e_{i}, W_{i}\right) \in D^{p}$ takes the form $r_{i}^{\prime}=\left(e i d_{i}, e_{i}, \sigma_{i}\right)$, for some $\sigma_{i} \in \mathcal{S}$ in $D^{*}$. By enumerating all such possible combinations we get the complete set of possible worlds. Assuming that the distributions associated with each record $r_{i}$ in $D^{p}$ are stochastically independent, the probability of a possible world $D^{*}$ is $\operatorname{Pr}\left[D^{*}\right]=\prod_{i=1}^{n} \operatorname{Pr}_{W_{i}}\left[\sigma_{i}\right]$.

Table 1. A source-level uncertain database (L) and one possible world $D^{*}(\mathrm{R})$ showing sources and associated events (here, $\operatorname{Pr}\left[D^{*}\right]=0.6 \times 0.3 \times 0.7=0.126$ ).

\begin{tabular}{|c|c|c|}
\hline eid & event & $W$ \\
\hline$e_{1}$ & $\mathrm{a}$ & $\left(\sigma_{1}: 0.6\right)\left(\sigma_{2}: 0.4\right)$ \\
\hline$e_{2}$ & $\mathrm{~b}$ & $\left(\sigma_{1}: 0.3\right)\left(\sigma_{2}: 0.2\right)\left(\sigma_{3}: 0.5\right)$ \\
\hline$e_{3}$ & $\mathrm{c}$ & $\left(\sigma_{1}: 0.7\right)\left(\sigma_{3}: 0.3\right)$ \\
\hline
\end{tabular}

\begin{tabular}{|c|l|}
\hline source & event $(\mathrm{s})$ \\
\hline$\sigma_{1}$ & $(a)(b)(c)$ \\
\hline$\sigma_{2}$ & \langle\rangle \\
\hline$\sigma_{3}$ & \langle\rangle \\
\hline
\end{tabular}

Table 2. An event-level uncertain database (L), all possible worlds for $D_{2}^{p}(\mathrm{C})$ and a possible world $D^{*}$ for $D^{p}(\mathrm{R})$ containing one world each from possible worlds of every $D_{i}^{p}$. (here, $\operatorname{Pr}\left[D^{*}\right]=0.126 \times 0.48 \times 0.35=0.021$ ).

\begin{tabular}{|c|c|}
\hline & p-sequence \\
\hline$D_{1}^{p}$ & $(a: 0.6)(b: 0.3)(c: 0.7)$ \\
\hline$D_{2}^{p}$ & $(a: 0.4)(b: 0.2)$ \\
\hline$D_{3}^{p}$ & $(b: 0.5)(c: 0.3)$ \\
\hline
\end{tabular}

\begin{tabular}{|c|c|}
\hline\langle\rangle & $0.6 \times 0.8=0.48$ \\
\hline$(a)$ & $0.4 \times 0.8=0.32$ \\
\hline$(b)$ & $0.6 \times 0.2=0.12$ \\
\hline$(a)(b)$ & $0.4 \times 0.2=0.08$ \\
\hline
\end{tabular}

\begin{tabular}{|c|l|}
\hline source & possible world \\
\hline$\sigma_{1}$ & $(a)(b)(c)=0.126$ \\
\hline$\sigma_{2}$ & \langle\rangle$=0.48$ \\
\hline$\sigma_{3}$ & \langle\rangle$=0.35$ \\
\hline
\end{tabular}

Event-Level Uncertainty. In some cases, the 'source' of the event is known but the 'event' itself is uncertain. Consider a scenario where employees movements are tracked in a building using RFID sensors [5]. A typical relation SIGHTING ( $t$, 
tID, aID) in PEEX system [5], denotes that the RFID tag tID was detected by antenna aID at time $t$. Consequently, PEEX processes the SIGHTING relation to output a higher-level uncertain relation such as MEETING(time, person1, person2, room, prob). An example tuple such as (103, 'Alice', 'Bob', 435, 0.4) in MEETING means that at time 103, PEEX believes that Alice and Bob are having a meeting (event) with probability 0.4 in room 435 (source) [5]; since antennae are at fixed locations, the source is certain but the event is uncertain.

A probabilistic database $D^{p}$ is a collection of p-sequences $D_{1}^{p}, \ldots, D_{m}^{p}$, where $D_{i}^{p}$ is associated with source $i \in \mathcal{S}, D_{i}^{p}=\left\langle\left(e_{1}, c_{1}\right) \ldots\left(e_{k}, c_{k}\right)\right\rangle$, where the events $e_{j}$ are ordered by eid and $c_{j}$ is the confidence that $e_{j}$ actually occurred. The possible worlds semantics of $D^{p}$ is as follows. For each event $e_{j}$ in a p-sequence $D_{i}^{p}$ there are two kinds of worlds; one in which $e_{j}$ occurs and the other where it does not. Let occurred $=\left\{x_{1}, \ldots, x_{l}\right\}$, where $1 \leq x_{1}<\ldots<x_{l} \leq k$, be the indices of events that occur in $D_{i}^{*}$. Then $D_{i}^{*}=\left\langle e_{x_{1}}, \ldots, e_{x_{l}}\right\rangle$, and $\operatorname{Pr}\left(D_{i}^{*}\right)=$ $\prod_{j \in \text { occurred }} c_{j} * \prod_{j \notin \text { occurred }}\left(1-c_{j}\right)$. The set of all possible worlds of $D_{i}^{p}$, denoted by $P W\left(D_{i}^{p}\right)$ is obtained by taking all possible $2^{l}$ alternatives for occurred, and we say $P W\left(D^{p}\right)=P W\left(D_{1}^{p}\right) \times \ldots \times P W\left(D_{m}^{p}\right)$. For any $D^{*} \in P W\left(D^{p}\right)$ such that $D^{*}=\left(D_{1}^{*}, \ldots, D_{m}^{*}\right)$, the probability of $D^{*}$ is given by: $\operatorname{Pr}\left[D^{*}\right]=\prod_{i=1}^{m} \operatorname{Pr}\left(D_{i}^{*}\right)$.

\section{$3 \quad$ Probabilistic Frequentness}

For Frequent itemset mining in probabilistic databases, measures like expected support [1] and probabilistic frequentness [3] have been used. An expected support based approach for mining sequential patterns in probabilistic databases was proposed in [6]. Here, we focus on probabilistic frequent sequential patterns.

Definition 1. Given a probabilistic database $D^{p}$ and its set of possible worlds $P W\left(D^{p}\right)$, the support probability for a sequence $s$ is denoted by: $\operatorname{Pr}_{i}(s)=$ $\sum_{D^{*} \in P W\left(D^{p}\right),\left(\operatorname{Supp}\left(s, D^{*}\right)=i\right)} \operatorname{Pr}\left(D^{*}\right)$, where $\operatorname{Supp}\left(s, D^{*}\right)$ is the support of $s$ in $D^{*}$. Note that $\operatorname{Pr}_{i}(s)$ is the probability that the support of $s$ is exactly $i$. Further, define the support probability distribution $(S P D)$ as the vector $\left\langle\operatorname{Pr}_{0}(s), \ldots, \operatorname{Pr}_{m}(s)\right\rangle$.

Denote by $\operatorname{Pr}_{\geq \theta}(s)=\sum_{k=\theta}^{m} \operatorname{Pr}_{k}(s)$ the probability that the support of $s$ is at least $\theta$. Given $D^{p}$ and two user-specified thresholds namely support $\theta, 1 \leq \theta \leq m$ and a confidence $\tau \in(0,1]$, the objective is to find all probabilistic frequent sequences (PFSes) $s$ s.t. $\operatorname{Pr}_{\geq \theta}(s) \geq \tau$ (i.e. all $s$ with probability $\geq \tau$ of having support $\geq \theta)$. Next, we show that we can obtain PFSes by dynamic programming (DP) for event-level uncertainty. By contrast, we show the computational intractability of finding PFSes for source-level uncertainty. We consider the fundamental question "is $s$ a PFS", i.e. given $D^{p}, s, \theta$ and $\tau$, is $\operatorname{Pr}_{\geq \theta}(s) \geq \tau$ ?

PFSes for Event-Level Uncertainty. First, we compute the probability with which a source supports a sequence $s$ i.e. we compute $\operatorname{Pr}\left(s \preceq D_{i}^{p}\right) \forall i, 1 \leq i \leq m$, as done by [6]. Then, we compute $\operatorname{Pr}_{i, j}(s)$, for $0 \leq i, j \leq m$, which is the probability that exactly $i$ of the first $j$ sources support $s$, by DP using the recurrence:

$$
\operatorname{Pr}_{i, j}(s)=\operatorname{Pr}_{i-1, j-1}(s) \cdot \operatorname{Pr}\left(s \preceq D_{i}^{p}\right)+\operatorname{Pr}_{i, j-1}(s) \cdot\left(1-\operatorname{Pr}\left(s \preceq D_{i}^{p}\right)\right),
$$


where $\operatorname{Pr}_{0, j}(s)=1,0 \leq j \leq m$ and $\operatorname{Pr}_{i, j}(s)=0, \forall i>j$. Clearly, $\operatorname{Pr}_{i, m}(s)=$ $\operatorname{Pr}_{i}(s)$, for all $i$, and we can use this to determine if $s$ is a PFS.

PFSes for Source-Level Uncertainty. In source-level uncertainty, an event may potentially be associated to more than one sources as shown in Table 1. Note that the DP computation in Eq. 1 computes the value $\operatorname{Pr}_{i, j}(s)$, which does not help in this case. For example, in Table 1, event 'b' is confused between sources $\sigma_{1}, \sigma_{2}$ and $\sigma_{3}$, but it could only be associated to one of the three in a real world, which is ignored when using Eq. 1. For example, for $s=(b), \operatorname{Pr}_{2,2}(s)=0$, as only one of the sources can support $s$. However, using Eq. 1, we obtain: $\operatorname{Pr}_{2,2}(s)=$ $\operatorname{Pr}_{1,1} \times \operatorname{Pr}\left(s \preceq D_{2}^{p}\right)+\operatorname{Pr}_{2,1} \times\left(1-\operatorname{Pr}\left(s \preceq D_{2}^{p}\right)\right)=0.3 \times 0.2+0 \times 0.8=0.06$, which is not correct. So, Eq. 1 does not work for source-level uncertainty. We further note that it is not possible to compute the value $\operatorname{Pr}_{i, j}(s)$. As mentioned above that $\operatorname{Pr}_{k, m}(s)=\operatorname{Pr}_{k}(s)$, for all $k$, we say that computing $\operatorname{Pr}_{k}(s)$ (i.e. the probability that exactly $k$ sources support $s)$ as Exact-k-Support problem.

Theorem 1. Given a probabilistic database $D^{p}$, a sequence $s$ and a number $k, 0 \leq k \leq m$, computing the Exact-k-Support for $s$ in $D^{p}$ is $\sharp P$-complete.

Theorem 1 is shown by reducing the problem of computing the number of perfect matchings in a bipartite graph, a $\sharp \mathrm{P}$ complete problem [8], to the Exactk-Support problem.

\section{Conclusions and Future Work}

We studied uncertainty models for sequential pattern mining and discussed probabilistic frequentness computation for source-level and event-level uncertainties. An empirical evaluation and comparison with expected support in computational cost and in quality of the solution should be an interesting direction to explore.

\section{References}

1. Aggarwal, C.C. (ed.): Managing and Mining Uncertain Data. Springer (2009)

2. Agrawal, R., Srikant, R.: Mining sequential patterns. In: ICDE. pp. 3-14 (1995)

3. Bernecker, T., Kriegel, H.P., Renz, M., Verhein, F., Züfle, A.: Probabilistic frequent itemset mining in uncertain databases. In: KDD. pp. 119-128 (2009)

4. Hassanzadeh, O., Miller, R.J.: Creating probabilistic databases from duplicated data. The VLDB Journal 18(5), 1141-1166 (2009)

5. Khoussainova, N., Balazinska, M., Suciu, D.: Probabilistic event extraction from rfid data. In: ICDE. pp. 1480-1482 (2008)

6. Muzammal, M., Raman, R.: Mining sequential patterns from probabilistic databases. Tech. Rep. CS-10-002, Dept. of Computer Science, Univ. of Leicester (2010), available from http://www.cs.le.ac.uk/people/mm386/pSPM.pdf

7. Suciu, D., Dalvi, N.N.: Foundations of probabilistic answers to queries. In: SIGMOD Conference. p. 963 (2005)

8. Valiant, L.G.: The complexity of computing the permanent. Theor. Comput. Sci. 8, 189-201 (1979)

9. Wikipedia: http://en.wikipedia.org/wiki/anpr — Wikipedia, the free encyclopedia (2010), \{http://en.wikipedia.org/wiki/ANPR\}, [accessed 30-April-2010] 